\title{
OPEN Jasmonic acid and ERF family genes are involved in chilling sensitivity and seed browning of pepper fruit after harvest
}

\begin{abstract}
Jeong Gu Lee ${ }^{1}$, Gibum $\mathrm{Yi}^{1,2}$, Jieun Seo ${ }^{1}$, Byoung-Cheorl Kang ${ }^{1,2}$, Jeong Hee Choi ${ }^{3}$ \& Eun Jin Lee Led $^{1,4}$

Pepper (Capsicum annuum L.) fruit is sensitive to temperatures below $10^{\circ} \mathrm{C}$, which severely diminish fruit quality during cold chain distribution. Seed browning was a major chilling symptom in 36 genotypes of $C$. annuum fruit screened after storage at $2{ }^{\circ} \mathrm{C}$ for 3 weeks. Among them, pepper fruits of chilling-insensitive 'UZB-GJG-1999-51' and -sensitive 'C00562' were treated at $2{ }^{\circ} \mathrm{C}$ for 0 or $24 \mathrm{~h}$, respectively. Analyses of integrated transcriptome-metabolome and relative gene expression in seeds obtained from the two genotypes were conducted to identify key factors involved in the seed browning induced by chilling. The relative contents of branched-chain amino acids such as leucine, isoleucine, and valine were significantly increased after chilling. Transcriptome identification showed 3,140 differentially expressed genes (log twofold change $>1.0$ and FDR-corrected $p$ value $<0.05$ ) affected by chilling between the two genotypes. Particularly, genes related to jasmonic acid synthesis and signaling were differentially expressed. A regulatory network of jasmonic acid synthesis and signaling, and regulation of ERF family genes might contribute to chilling response in pepper fruit. The results of this study may help facilitate further studies to develop chilling-insensitive peppers and could be a basis for improving postharvest fruit quality.
\end{abstract}

Harvested crops are usually stored at low temperatures during the distribution and sale processes to maintain their freshness. However, pepper (Capsicum annuum L.) fruit, a subtropical crop, is sensitive to low temperatures. Various chilling symptoms appear at storage temperatures below $7-10^{\circ} \mathrm{C}$ depending on the variety, fruit maturity, and time of exposure to chilling temperatures ${ }^{1}$. Typical chilling symptoms in pepper fruit are; seed browning or discoloration, pitting on the surface, calyx discoloration, and shrinkage ${ }^{2}$. These injuries lower the marketability of pepper fruit during cold chain distribution.

Chilling symptoms have been known to result from the deterioration of cellular membranes by reactive oxygen species (ROS), mostly derived from the lipid peroxidation process ${ }^{3}$. In addition, jasmonic acid (JA) and salicylic acid (SA)-synthesis and -signaling pathways have been known to be involved in protecting against chilling stress in plants ${ }^{4,5}$. JA is involved in the chilling stress mechanism and it regulates chilling tolerance ${ }^{6,7}$. An increase in endogenous JA content under chilling stress in Arabidopsis ${ }^{8}$, tomato ${ }^{9}$, and pepper fruit ${ }^{5}$ has been reported. JA is synthesized from a-linolenic acid and lipoxygenase (LOX), allene oxide synthase (AOS), and allene oxide cyclase (AOC), which are enzymes involved in JA biosynthesis ${ }^{10}$. In order for JA to have biological activity, it conjugates with isoleucine to form jasmonoyl-isoleucine (JA-Ile), a JA active derivative; jasmonate resistant 1 (JAR1) is involved to form JA-Ile.

In the JA signaling pathway, one of the most important proteins is JASMONAT-ZIM-domain (JAZ), which acts as a repressor for JA signaling. If JA is absent or present in low levels, JAZ protein binds to downstream transcription factors such as MYC ${ }^{11}$, DELLA $^{12}$, and the inducer of C-repeat binding factor expression (ICE) ${ }^{8}$. JAZ protein limits the transcription factor's activities; however, in the presence of JA-Ile, JAZ protein is broken down by $26 \mathrm{~S}$ proteasome, and then transcription factors for the expression of downstream genes that are needed for the stress response pathway are activated ${ }^{13}$. CORONATINE INSENSITIVE 1 (COI1), which belongs to the

\footnotetext{
${ }^{1}$ Depatment of Agriculture, Forestry and Bioresources, College of Agriculture and Life Sciences, Seoul National University, Seoul 08826, Republic of Korea. ${ }^{2}$ Plant Genomics and Breeding Institute, Seoul National University, Seoul 08826, Republic of Korea. ${ }^{3}$ Korea Food Research Institute, Wanju-gun, Jeollabuk-do 55365, Republic of Korea. ${ }^{4}$ Research Institute of Agriculture and Life Sciences, Seoul National University, Seoul 08826, Republic of Korea. ${ }^{\circledR}$ email: ejinlee3@snu.ac.kr
} 
group of F-box proteins, binds to JAZ protein. Further, JA-Ile can bind to the COI1-JAZ complex to promote ubiquitination and degradation of JAZ protein. JA-Ile has been known to be required for COI1-mediated degradation of JAZ protein ${ }^{14}$.

Activation of apetala2/ethylene responsive factors (AP2/ERFs) such as ERF $1{ }^{15}$, ERF32 $2^{16}$, and ORA59 ${ }^{17}$ could begin in the presence of JA as well as under JAZ degradation. AP2/ERFs consist of two subfamilies: the inducer of the C-repeat binding factor/dehydration responsive element binding factor (CBF/DREB) and the ERF subfamily binding to GCC-box; both are involved in JA, SA, and ethylene responses to environmental stress. AP2/ERFs are important regulators for defense against abiotic stresses ${ }^{18}$. In particular, AP2/ERFs are known to be involved in cold stress in plants, and it has been reported that AP2/ERFs play a major role in chilling tolerance and cold acclimation in Arabidopsis ${ }^{19}$. However, the regulatory mechanism of AP2/ERFs in cold stress response is still unclear. In a recent study, it was reported that AP2/ERFs regulate the peroxidase-encoding genes ${ }^{20}$ WRKY $33^{21}$ and $\mathrm{CBF} / \mathrm{DREB}^{22}$, and mitigate reactive oxygen in response to cold stress.

The CBF/DREB subfamily binds to the dehydration-responsive element/C-repeat (DRE/CRT) elements and is regulated by the ICE transcription factor. As mentioned earlier, JAZ protein is known as a regulator of chilling stress because it suppresses the ICE transcription factor that is responsible for the chilling stress response. However, at chilling temperatures, an increased JA-Ile level activates ICE by the degradation of the COI1-JAZ complex $^{23}$. Then, ICE regulates CBF/DREB to contribute to chilling tolerance through CBF regulons such as responsiveness to desiccation genes and cold-responsive genes ${ }^{24}$. Therefore, many members of the CBF/DREB subfamily are involved in improving the stress tolerance of various plants under the environmental stresses of cold, drought, and salinity.

To understand the underlying mechanism of seed browning induced by chilling, we first screened seed browning occurrences of 36 genotypes of $C$. annuum fruit. We also investigated the metabolomics and transcriptomics of two pepper genotypes that show great differences in their occurrence rates of seed browning as a chilling symptom when exposed to chilling temperatures after harvest. Our results showed JA synthesis, JA signaling, and ERF family genes contribute to chilling response in pepper fruit. This study can help elucidate the cellular mechanism or identify key factors affecting chilling sensitivity or insensitivity of peppers after harvest. Also, this study provides information for further studies to develop chilling-insensitive pepper fruit and could be a basis for improving the postharvest fruit quality of peppers.

\section{Results}

Seed browning rates of 36 pepper genotypes. Seed browning symptoms were the major chilling injury in the 36 genotypes of $C$. annuum screened in this study (Supplementary Table S1). Other chilling symptoms such as pitting on the surface and discoloration of the calyx were negligible during cold storage at $2{ }^{\circ} \mathrm{C}$ for 3 weeks. Seed browning was also observed as a major chilling symptom of C. annuum 'Cheongyang' fruit treated at $2{ }^{\circ} \mathrm{C}$ for 3 weeks ${ }^{5}$. We sorted 36 pepper genotypes according to seed browning rates from $0.0 \%$ (chillinginsensitive peppers) to over $60.0 \%$ (chilling-sensitive peppers) (Supplementary Table S1).

During cold storage at $2{ }^{\circ} \mathrm{C}$ for 3 weeks, there were great differences in seed browning rates between the chilling-insensitive 'UZB-GJG-1999-51' and chilling-sensitive 'C00562' (Fig. 1A and Supplementary Table S1). The seed browning rate of chilling-sensitive ' $C 00562$ ' was $63.86 \%$, whereas the seed browning rate of chillinginsensitive ' $U Z B-G J G-1999-51$ ' was $0.00 \%$. Additionally, the seed browning rates of other chilling-insensitive genotypes 'Takanotsume' and 'Hungarian Wax', and other chilling-sensitive genotypes 'Chili bangi' and 'Gyeonggiyangpyeong' were in the order of $0.00 \%, 4.31 \%, 30.88 \%$, and $41.01 \%$, respectively (Supplementary Fig. S1 and Supplementary Table S1).

Differentially accumulated metabolites. A total 51 of untargeted and targeted metabolites in pepper seeds were quantified in this study. To confirm the relationships among identified metabolites, partial least squares-discriminant analysis (PLS-DA) was performed (Fig. 1B,C). The first and second principle components (PCs) accounted for 47.6\% (PC 1, 34.6\%; PC 2, 13.0\%) of the variance in the dataset. The PLS-DA loading plot (Fig. 1C) was used to identify the causative metabolites of the PLS-DA score plot. We were able to confirm that pyruvic acid, lactic acid, oxalic acid, palmitic acid, and methionine were the major metabolites separating the two pepper genotypes (chilling-insensitive ' $U Z B-G J G-1999-51$ ' and chilling-sensitive 'C00562'). In addition, among amino acids, isoleucine, valine, and leucine were the major metabolites contributing to the distribution between ' $0 \mathrm{~h}$ ' and ' $24 \mathrm{~h}$ ' pepper groups, with separation due to the chilling treatment at $2{ }^{\circ} \mathrm{C}$ for $24 \mathrm{~h}$.

Figure $2 \mathrm{~A}$ shows the heat-map analysis of metabolites. Most organic acids, except for malic acid and citric acid, showed substantially higher contents in chilling-sensitive 'C00562' than in chilling-insensitive ' $U Z B$ GJG-1999-51'. Also, leucine, valine, and isoleucine contents were clearly increased in the two pepper genotypes by chilling treatment; however, chilling-insensitive ' $U Z B-G J G-1999-51$ ' had substantially higher contents of leucine, valine, and isoleucine than chilling-sensitive 'C00562'.

The relative content levels of 10 selected metabolites from organic acids, amino acids, and fatty acids are compared in Fig. 2B. The levels of pyruvic acid, succinic acid, and fumaric acid were substantially higher in chilling-sensitive 'C00562', regardless of chilling treatment, at $2{ }^{\circ} \mathrm{C}$ for $24 \mathrm{~h}$. The pyruvic acid level was significantly increased in 'C00562' only after chilling treatment. In the cases of leucine, isoleucine, and valine, which are branched-chain amino acids (BCAA), there were no differences in their contents between the two pepper genotypes, and they showed similar levels to each other at $0 \mathrm{~h}$, before chilling treatment. However, after chilling treatment $(24 \mathrm{~h})$, the three amino acid levels were significantly increased in the two pepper genotypes.

The level of palmitic acid was not significantly changed in chilling-insensitive ' $U Z B-G J G-1999-51$ ' by chilling treatment, but it was significantly increased in chilling-sensitive 'C00562'. Citric acid and malic acid levels were not significantly different. Linoleic acid content was slightly higher in chilling-insensitive ' $U Z B-G J G-1999-51$ ' 
A

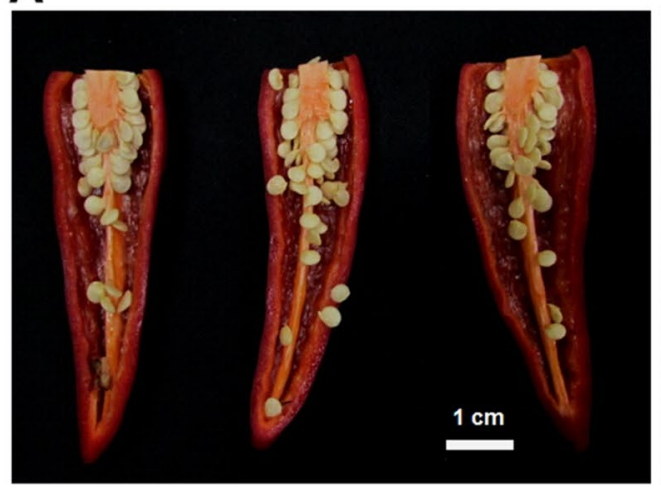

'UZB-GJG-1999-51' (chilling insensitive, Ins)

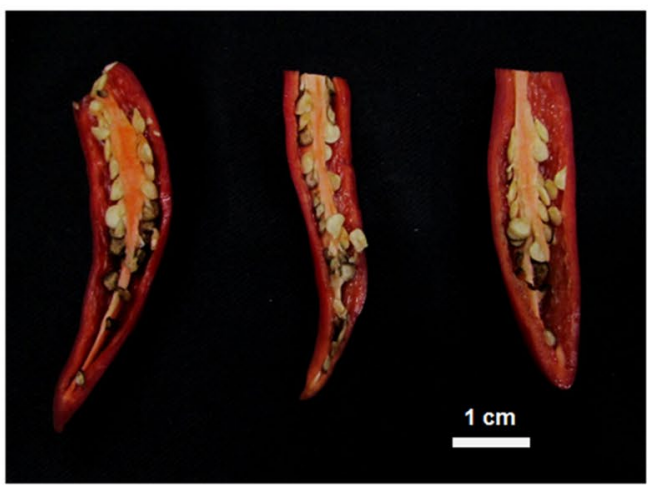

'C00562' (chilling sensitive, Sen)
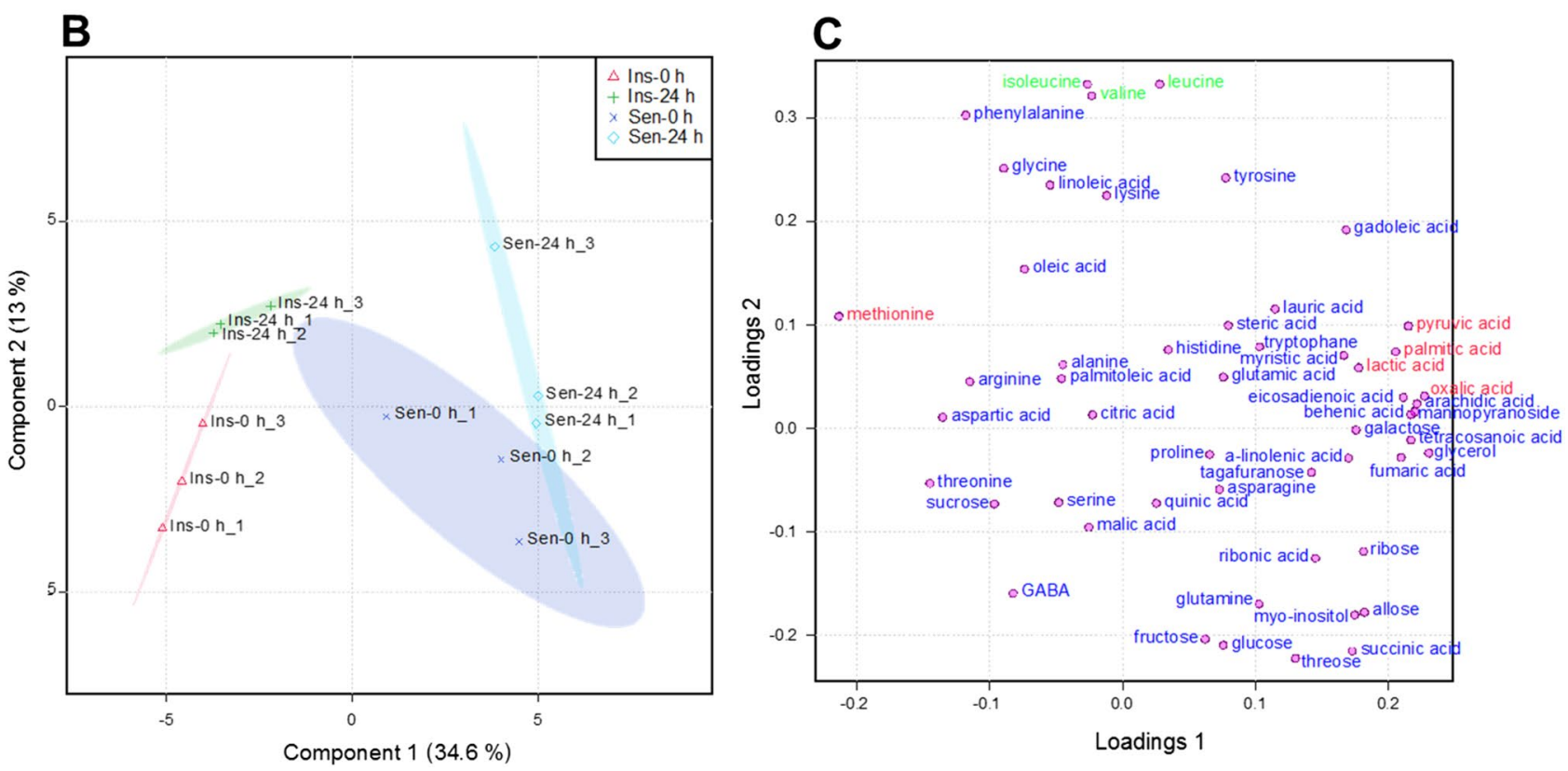

Figure 1. Seed browning and metabolite analysis (A-C). Seed browning appearances (A) of ' $U Z B$ GJG-1999-51' (chilling-insensitive, Ins) and 'C00562' (chilling-sensitive, Sen) peppers induced by cold storage at $2{ }^{\circ} \mathrm{C}$ for 3 weeks. Analysis of PLS-DA score plot (B) and loading plot (C) of metabolites in seeds obtained from both peppers after chilling treatment at $2^{\circ} \mathrm{C}$ for $0 \mathrm{~h}$ and $24 \mathrm{~h}$, respectively. $(\mathbf{B}, \mathbf{C})$ were created with freely available MetaboAnalyst (version 3.0, https://www.metaboanalyst.ca/).

than in chilling-sensitive 'C00562' but there were no significant differences according to chilling sensitivity of genotypes or according to the chilling treatment at $2{ }^{\circ} \mathrm{C}$ for $24 \mathrm{~h}$.

To reveal the biological pathways involved in a chilling-induced seed browning in peppers by chilling treatment at $2{ }^{\circ} \mathrm{C}$ for $24 \mathrm{~h}$, metabolite enrichment was analyzed (Fig. 3A). As a result, galactose metabolism; valine, leucine, and isoleucine biosynthesis; phenylalanine, tyrosine, and tryptophan biosynthesis; and starch and sucrose metabolism pathways in peppers were determined to be significantly affected by the chilling treatment at $2{ }^{\circ} \mathrm{C}$ for $24 \mathrm{~h}$.

Differentially expressed genes (DEGs). Through the transcriptome analysis of seeds obtained from chilling-insensitive ' $U Z B-G J G$-1999-51' and chilling-sensitive 'C00562' before or after chilling treatment, an average of 23.9 million reads ranging from 15.1 to 36.8 million reads among 12 samples (two genotypes + before and after chilling +3 biological replicates) were obtained. On average, $82.7 \%$ of total reads were mapped to the transcript library. Furthermore, the percentages of Q30 base in all samples were higher than $96.50 \%$ and the percentages of Q20 were higher than 98.03\% (Supplementary Table S3).

In total, 84,627 contigs were assembled with a reference genome (Pepper_Zunla_1_Ref_v1.0_rna.fna, accession number: GCF_000710875.1). The average contig length was $1010.6 \mathrm{bp}$, and the N50 was $1518 \mathrm{bp}$. To confirm the relationships among each sample and replicate, PCA and PLS-DA were performed using the changes 


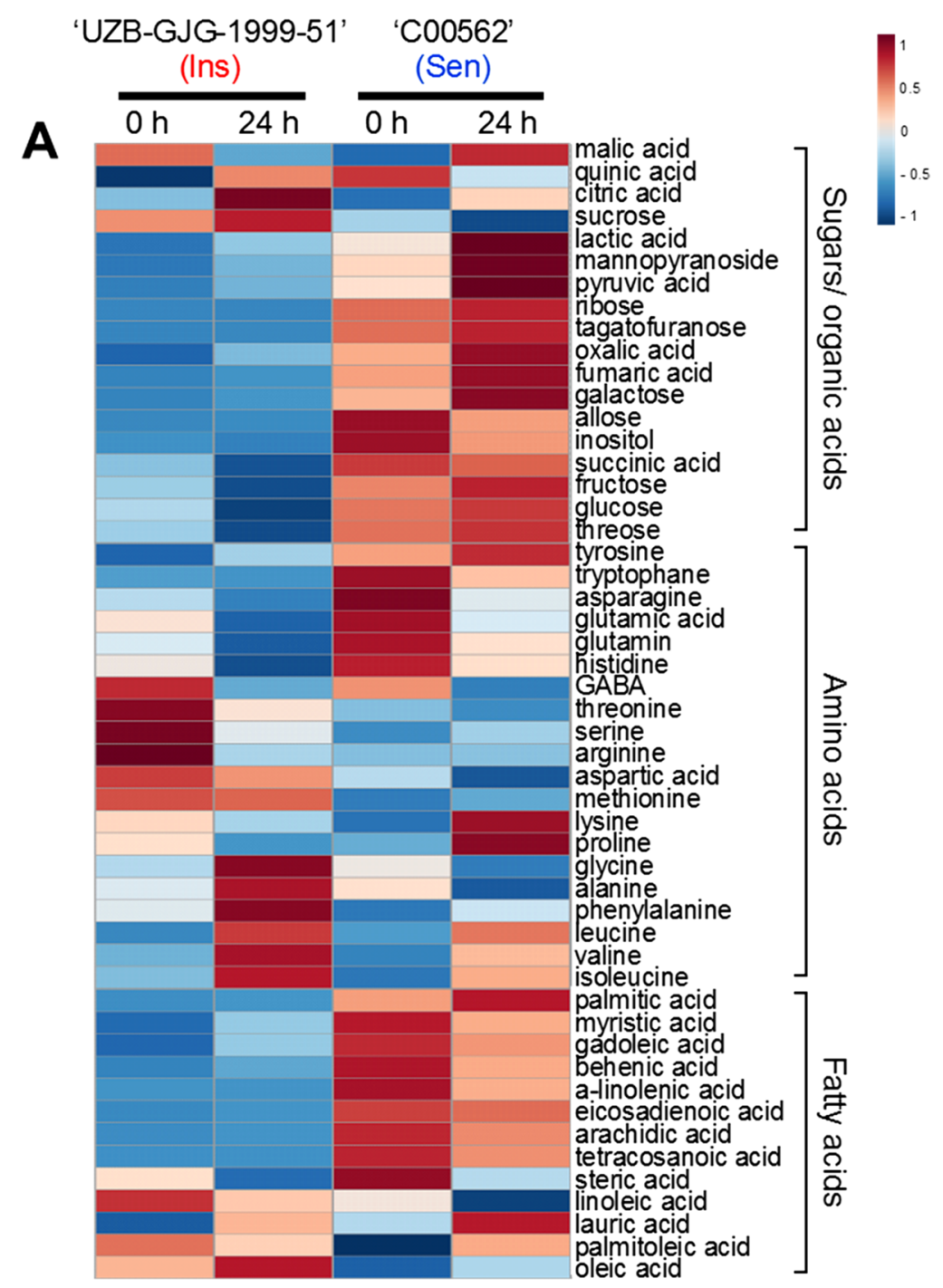

B
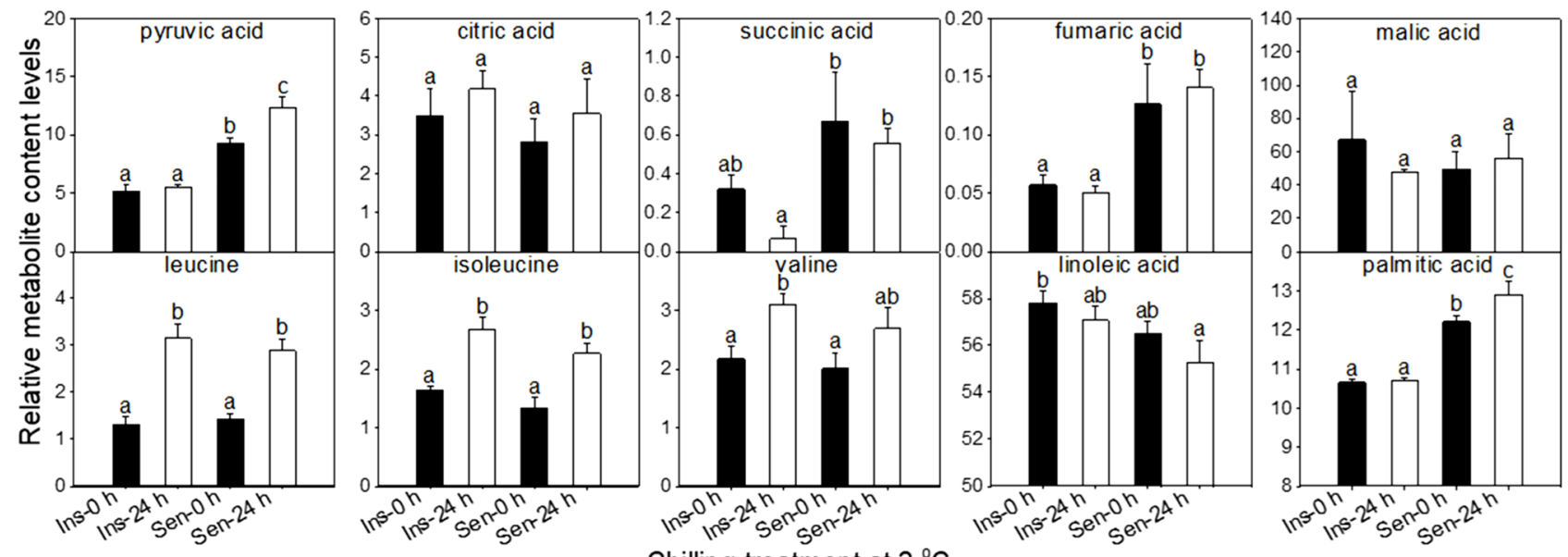

Chilling treatment at $2{ }^{\circ} \mathrm{C}$

Figure 2. Differentially accumulated metabolites (DAMs) (A, B). Heat-map analysis (A) of DAMs and the relative metabolite levels (B) of 10 selected organic acids, amino acids, and fatty acids of seeds obtained from 'UZB-GJG-1999-51' (chilling-insensitive, Ins)' and 'C00562' (chilling-sensitive, Sen) peppers after chilling treatment at $2^{\circ} \mathrm{C}$ for $0 \mathrm{~h}$ and $24 \mathrm{~h}$, respectively. Vertical bars are means $\pm S D(n=3)$. Different letters represent significant differences $(p<0.05)$ in Duncan's multiple range test. $(\mathbf{A})$ was created with freely available MetaboAnalyst (version 3.0, https://www.metaboanalyst.ca/). 
A

valine galactose metabolism valine, leucine and isoleucine biosynthesis starch and sucrose metabolism phenylalanine metabolism
alanine, aspartate and glutamate metabolism arginine biosynthesis glyoxylate and dicarboxylate metabolism glyc ine, serine and threonine metabolism fructose and mannose metabolism valine, leucine and isoleucine degradation D-glutamine and D-glutamate metabolism nitrogen metabolism ascorbate and aldarate metabolism ubiquinone and other terpenoid-quinone biosynthesis cysteine and methionine metabolism arginine and proline metabolism histidine metabolism glycerolipid metabolism pantothenate and COA biosynthesis pentose phosphate pathway lysine degradatio glutathione metabolism inositol phosphate metabolism porphyrin and chlorophyll metabolism amino sugar and nucleotide sugar metabolism pyrimidine metabolism tryptophan metabolism tyrosine metabolism fatty acid biosynthesis purine metabolism

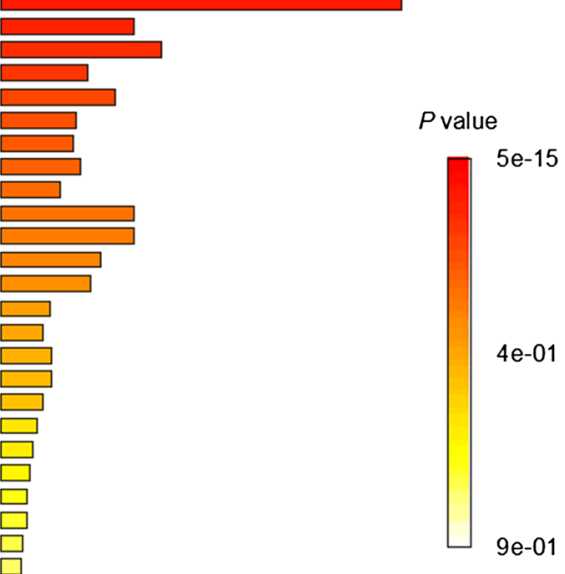

B

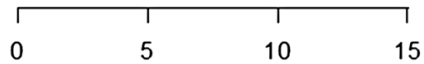

Fold enrichment

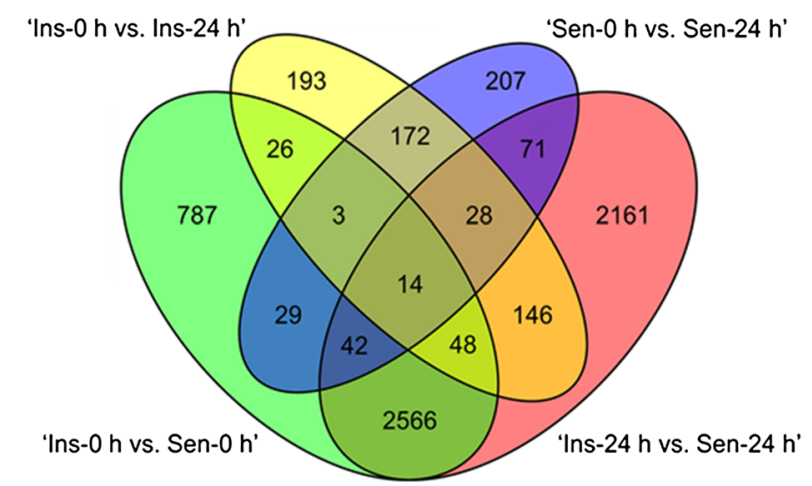

\section{C}

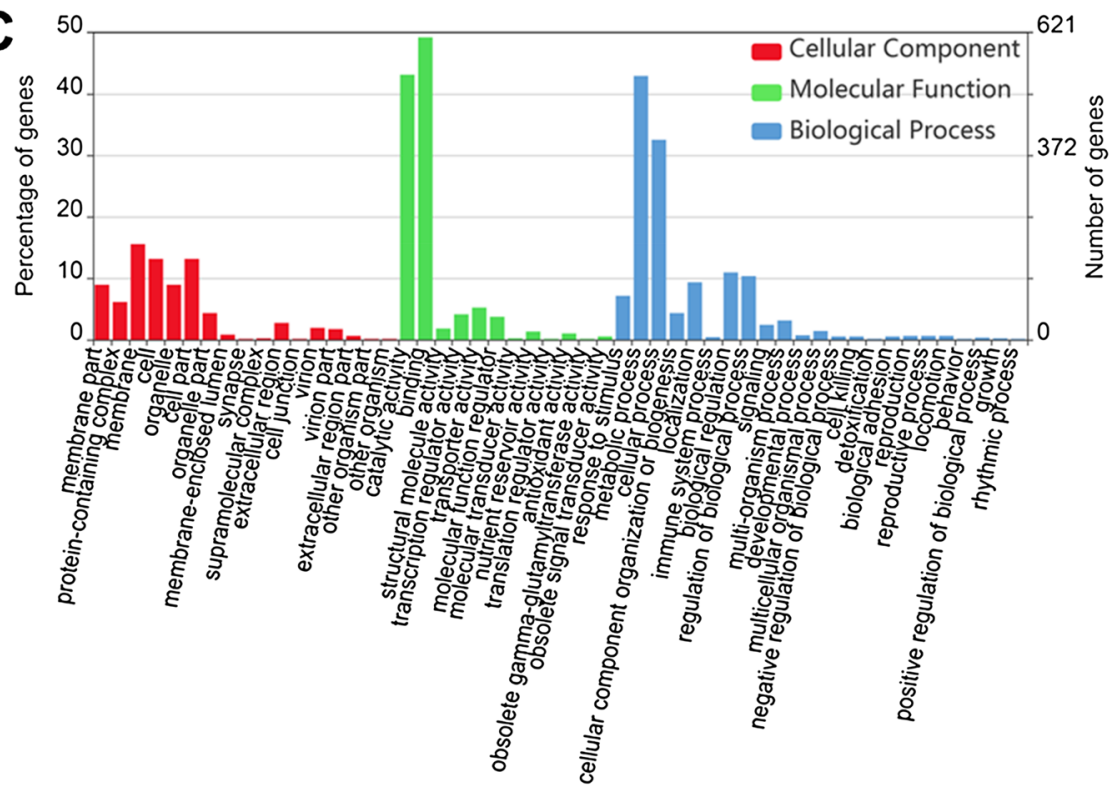

Figure 3. Metabolite set enrichment (MSE) and differentially expressed genes (DEGs) (A-C). MSE analysis (A), Venn diagrams of DEGs (B), and gene ontology annotation (C) of seeds obtained from 'UZB-GJG-1999-51' (chilling-insensitive, Ins) and 'C00562' (chilling-sensitive, Sen) peppers after chilling treatment at $2{ }^{\circ} \mathrm{C}$ for $0 \mathrm{~h}$ and $24 \mathrm{~h}$, respectively. The numbers marked in the diagram indicate the number of significantly up- and downregulated genes among the four DEG sets (log twofold change $>1.0$ and FDR-corrected $p$ value $<0.05$ ). (A) was created with freely available MetaboAnalyst (version 3.0, https ://www.metaboanalyst.ca/). 
of contigs (Supplementary Fig. S2). In the PCA score plot and PLS-DA score plot, the sum of first and second principal components accounted for $69.5 \%$ and $68.9 \%$ of the variance in the dataset, respectively. Also, the results of hierarchical analysis showed a clear distinction between the two pepper genotypes and the chilling treatment (Supplementary Fig. S3).

To minimize false positives, DEGs were selected using a threshold of $\geq$ twofold upregulated or downregulated genes with an FDR $<0.05$. As a result, a total of 6493 contigs were defined as DEGs of chilling-insensitive (Ins) 'UZB-GJG-1999-51' and chilling-sensitive (Sen) 'C00562' (Fig. 3B). Gene ontology (GO) term and Kyoto Encyclopedia of Genes and Genomes (KEGG) enrichment were performed using 3,140 DEGs to identify the differences in specific gene expressions induced by the chilling treatment at $2{ }^{\circ} \mathrm{C}$ for $24 \mathrm{~h}$. As an exception, 3353 contigs belong to only 'Ins- 0 h vs. Sen-0 h' (787 contigs), and only in an intersection between 'Ins- 0 h vs. Sen- 0 h' and 'Ins- 24 h vs. Sen- 24 h' (2566 contigs).

Upregulated or downregulated biological functions by the application of chilling treatment at $2{ }^{\circ} \mathrm{C}$ for $24 \mathrm{~h}$ were investigated with GO-based enrichment analysis (Fig. 3C). The top 5, significantly enriched GO terms in the cellular component category were 'intracellular (GO:0005622)', 'intracellular part (GO:0044424)', 'intracellular organelle (GO:0043229)', 'cell part (GO:0044464)', and 'membrane part (GO:0044425)'. Also, the top 5 enriched GO terms in the molecular function category were 'protein binding (GO:0005515)', 'small molecule binding (GO:0036094)', 'ion binding (GO:0043167)', 'organic cyclic compound binding (GO:0097159)', and 'transferase activity (GO:0016740)'. Additionally, 'primary metabolic process (GO:0044238)', 'nitrogen compound metabolic process (GO:0006807)', 'biosynthetic process (GO:0009058)', 'cellular metabolic process (GO:0044237)', and 'organic substance metabolic process (GO:0071704)' belonged to the biological process category.

Protein-protein interaction (PPI) network. DEGs were used to construct the PPI network which was composed of 760 nodes and 228 edges (Supplementary Fig. S4). Among them, 11 of the JA-related genes including LOX, AOC, JAR1, JAZ1, and JAZ3 were highlighted by a colored circle in Supplementary Fig. S4A. Also, except for PKT3, all of the JA-related genes were involved in abiotic, stress-related gene networks (Supplementary Fig. S4B). Therefore, it could be inferred that JA signaling is activated in the two genotypes; chilling-insensitive 'UZB-GJG-1999-51' and sensitive 'C00562'.

Heat-map of DEGs. To better understand the molecular responses of a chilling-induced seed browning of pepper fruit, a heat-map analysis was conducted using 29 DEGs consisting of 20 kinds of AP2/ERF family genes and 9 kinds of JA-related genes (Fig. 4 and Supplementary Table S4). Heat-map analysis clearly showed the differences in expression levels of 29 DEGs between chilling-insensitive 'UZB-GJG-1999-51' and chillingsensitive 'C00562' before and after chilling treatment at $2{ }^{\circ} \mathrm{C}$ for $0 \mathrm{~h}$ and $24 \mathrm{~h}$ (Fig. 4). Supplementary Fig. S5 shows the correlation and correlation network analysis of 51 metabolites and 29 DEGs of seeds obtained from 'UZB-GJG-1999-51' and 'C00562' peppers.

Among the 20 kinds of AP2/ERF families, except for CaDREB3 and CaERF11, the expression levels of CaERF106, CaRAP24, CaERF1, CaERF3_1, CaRAVL1, CaERF10, and CaERF5 were upregulated by chilling treatment at $2{ }^{\circ} \mathrm{C}$ in the two genotypes (Fig. 4). Their expression levels were more highly upregulated in chilling-insensitive ' $U Z B-G J G-1999-51^{\prime}$ ' than in chilling-sensitive 'C00562'. However, the expression levels of the other AP2/ERF families of CaERF3_2, CaERF80,CaAP2L1, CaERF4_1, CaERF4_3, CaERF91, CaERF114, and CaERF4_2 were upregulated in chilling-sensitive 'C00562' by the chilling treatment at $2{ }^{\circ} \mathrm{C}$, but they were not upregulated in chilling-insensitive 'UZB-GJG-1999-51'.

The expression level of CaJAR1, a JA-Ile biosynthesis gene, was up-regulated by chilling treatment in the two pepper genotypes, 'UZB-GJG-1999-51' and 'C00562' (Fig. 4). A higher CaJAR1 expression level was observed in chilling-insensitive ' $U Z B-G J G$-1999-51' than in chilling-sensitive 'C00562'. Overall expression trends of CaDREB3 and CaERF11 were opposite to the overall expression trends of CaJAR1, CaERF1, CaERF3_1, CaERF10, and CaERF5. The levels of CaDREB3 and CaERF11 were more highly expressed in chilling-sensitive 'C00562' than in chilling-insensitive ' $U Z B-G J G-1999-51^{\prime}$ and the expression levels of CaDREB3 and CaERF11 were downregulated by the chilling treatment at $2^{\circ} \mathrm{C}$ in both genotypes.

Relative gene expression levels. For quantitative PCR (qPCR), we analyzed seeds obtained from 6 additional pepper genotypes; 3 chilling-insensitive pepper fruits ('UZB-GJG-1999-51', 'Takanotsume', and 'Hungarian Wax'), and 3 chilling-sensitive pepper fruits ('C00562', 'Chili bangi', and 'Gyeonggiyangpyeong') (Supplementary Fig. S1 and Supplementary Table S1).

As shown in the results of the relative gene expression studies (Fig. 5), we could find clear differences between the chilling-insensitive and chilling-sensitive pepper fruits both before and after the chilling treatment. Analyzed genes were characterized into four categories: (1) genes that originally showed lower expression levels in insensitive genotypes were CaEFR11, CaDREB3, CaLOX2.1, and CaJAZ1; (2) genes that originally showed higher expression levels in insensitive genotypes were CaERF1, CaEFR3_1, CaERF5, CaERF10, and CaJAR1; (3) genes that showed significantly upregulated expression levels by chilling treatment were CaERF1, CaERF3_1, CaERF5, CaERF10, CaLOX1.5_2, CaAOS3, and CaJAR1; (4) conversely, genes that showed significantly downregulated expression levels by chilling treatment were CaERF11, CaDREB2, CaDREB3, CaLOX2.1, CaAOC, CaJAZ3, and CaAFP3.

The expression levels of transcription factors CaERF1, CaERF3_1, CaERF5, and CaERF10 were significantly upregulated by chilling treatment in the 6 pepper genotypes (Fig. 5 ). At $0 \mathrm{~h}$, before the chilling treatment, their expression levels were similar regardless of the genotypes. However, at $24 \mathrm{~h}$, after chilling treatment, their expression levels were substantially higher in the chilling-insensitive genotypes of 'UZB-GJG-1999-51', 'Takanotsume', and 'Hungarian Wax' than in the chilling-sensitive genotypes of 'C00562', 'chili bangi', and 'Gyeonggiyangpyeong.' 


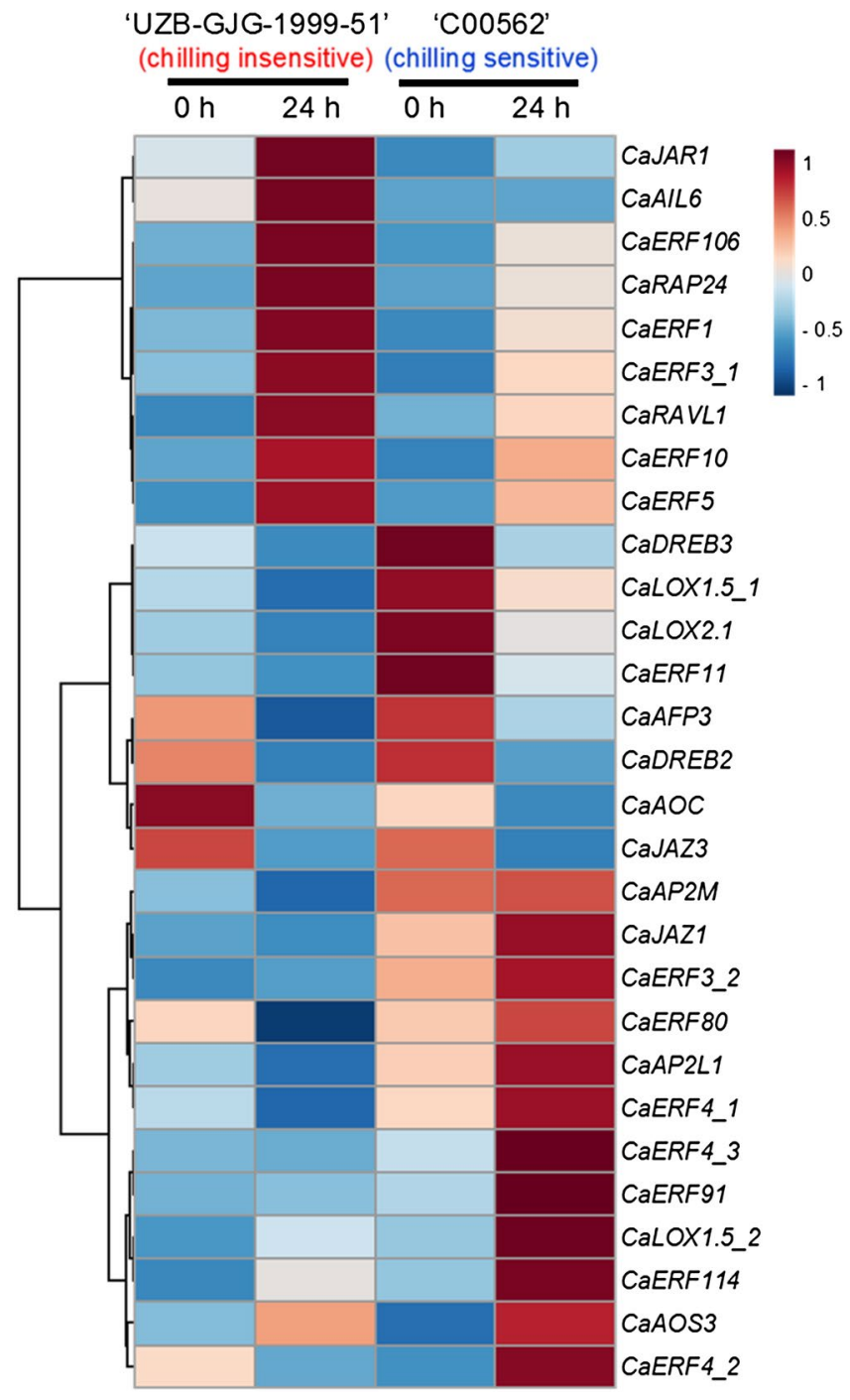

Figure 4. Heat-map of differentially expressed genes (DEGs). DEGs in jasmonic acid related genes and AP2/ ERF family genes of seeds obtained from ' $U Z B-G J G-1999-51$ ' (chilling-insensitive) and 'C00562' (chillingsensitive) peppers after chilling treatment at $2^{\circ} \mathrm{C}$ for $0 \mathrm{~h}$ and $24 \mathrm{~h}$, respectively. This figure was created with freely available MetaboAnalyst (version 3.0, https://www.metaboanalyst.ca/).

At 0 h, the relative expression levels of CaERF11, CaDREB3, and CaLOX2.1 were significantly higher in the chilling-sensitive genotypes than in the chilling-insensitive genotypes (Fig. 5). However, their expression levels were substantially down-regulated in the 6 pepper genotypes after chilling treatment for $24 \mathrm{~h}$. Chilling-insensitive genotypes showed substantially lower expression levels of CaERF11, CaDREB3, and CaLOX2.1 than chillingsensitive genotypes. Overall, their expression trends were very similar to each other, and the responses of their cellular mechanisms to chilling might be closely connected to each other in pepper fruit. We could assume that CaERF11 and CaDREB3 transcription factors act as negative regulators in the chilling response of pepper fruit.

CaLOX1.5_1 expression levels were not different, however. The expression levels of CaLOX1.5_2 were significantly upregulated in the chilling-insensitive and chilling-sensitive genotypes after chilling treatment (Fig. 5). The expression of CaLOX1.5_2 was more highly upregulated in chilling-sensitive genotypes than in insensitive genotypes. The expression levels of CaLOX1.5_2 showed similar levels in all peppers at $0 \mathrm{~h}$.

The CaJAR1 expressions were opposite to the CaERF11 and CaDREB3 expressions (Fig. 5). CaJAR1 expressions were significantly upregulated in chilling-insensitive genotypes by the chilling treatment, and were slightly upregulated in chilling-sensitive genotypes. Chilling-insensitive groups originally had higher CaJAR1 expressions than chilling-sensitive groups.

The levels of $C a D R E B 2, C a A O C, C a J A Z 3$, and CaAFP3 expressions were significantly higher in the 6 pepper genotypes at $0 \mathrm{~h}$, before the chilling treatment (Fig. 5). However, they were clearly downregulated after the chilling treatment. Contrary to the expression levels of CaDREB2, CaAOC, CaJAZ3, and CaAFP3, the expression level of $C a A O S 3$ was significantly upregulated by the chilling treatment but there were no differences according to the genotypes. The chilling treatment upregulated the expression level of CaJAZ1 only in chilling-sensitive groups, but not in chilling-insensitive groups. 


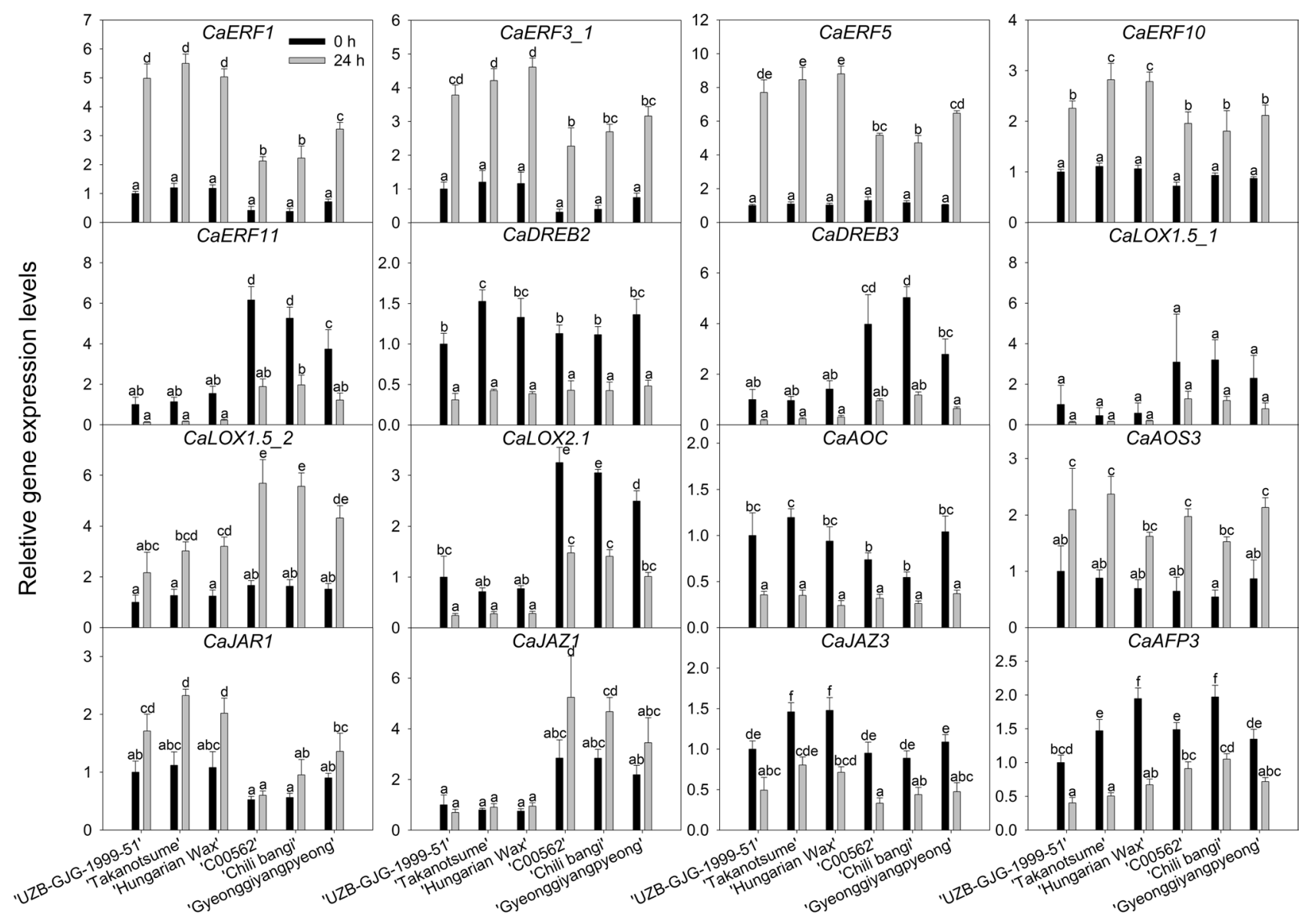

Figure 5. Relative expression levels of AP2/ERF family genes and jasmonic acid-related genes. Analysis of seeds obtained from chilling-insensitive 'UZB-GJG-1999-51', 'Takanotsume', 'Hungarian Wax' pepper fruit; and chilling-sensitive 'C00562', 'Chili baggi', and 'Gyeonggiyangpyeong' pepper fruit after chilling treatment at $2{ }^{\circ} \mathrm{C}$ for $0 \mathrm{~h}$ and $24 \mathrm{~h}$, respectively. Vertical bars are means $\pm \mathrm{SD}(\mathrm{n}=3)$. Different letters represent significant difference $(p<0.05)$ in Duncan's multiple range test. The seed browning rate of each genotype is shown in Supplementary Table S1.

Figure 6 shows the putative chilling response pathway predicted in C. annuum exposed to chilling conditions. Chilling-insensitive pepper fruit synthesizes more JA-Ile by higher upregulation of JAR1, and by higher rates of increasing isoleucine content than chilling-sensitive pepper fruit under chilling conditions. The transcription factors of CaERF1, CaERF3_1, CaERF5, and CaERF10 might act as positive regulators to increase the chilling tolerance by showing higher expression levels in chilling-insensitive pepper fruit than in chilling-sensitive pepper fruit. However, CaDREB3 and CaERF11 transcription factors might act as negative regulators by showing highly upregulated expression levels in sensitive pepper fruit before chilling as well as by showing downregulated expression levels after chilling.

\section{Discussion}

We analyzed a total of 51 targeted and untargeted metabolites of seeds obtained from chilling-insensitive ' $U Z B$ GJG-1999-51' and chilling-sensitive 'C00562' peppers exposed to $2{ }^{\circ} \mathrm{C}$ for $24 \mathrm{~h}$, and confirmed the changes of various metabolite levels (Fig. 1 and Fig. 2A). Our PLS-DA analysis indicated that pepper fruit are apparently separated according to their genotypes (PC 1); however, they are not clearly separated according to the chilling exposure time of $24 \mathrm{~h}$ (PC 2) (Fig. 1B). Metabolites are the end products of cell biology regulation processes and are closely related to phenotypes. Their levels can be regarded as the response of plant development to genetic and environmental changes ${ }^{25,26}$. Our results indicate that chilling-insensitive and sensitive pepper genotypes show differentially expressed metabolites, and that their levels are affected by chilling. We found that chilling exposure for $24 \mathrm{~h}$ was not enough to induce significant changes in all metabolites that were directly associated with the phenotype of seed browning induced by chilling.

The major differentially-expressed metabolites in the pepper fruit seeds were the organic acids; pyruvic acid, succinic acid, and fumaric acid. Their levels were significantly higher in chilling-sensitive 'C00562' peppers than in chilling-insensitive ' $U Z B-G J G-1999-51$ ' peppers. Particularly, the pyruvic acid content was only significantly increased in chilling-sensitive 'C00562' peppers by the chilling treatment (Fig. 2A,B). According to a previous study, pyruvic acid was highly accumulated in cucumber and eggplant which were exposed to a chilling 


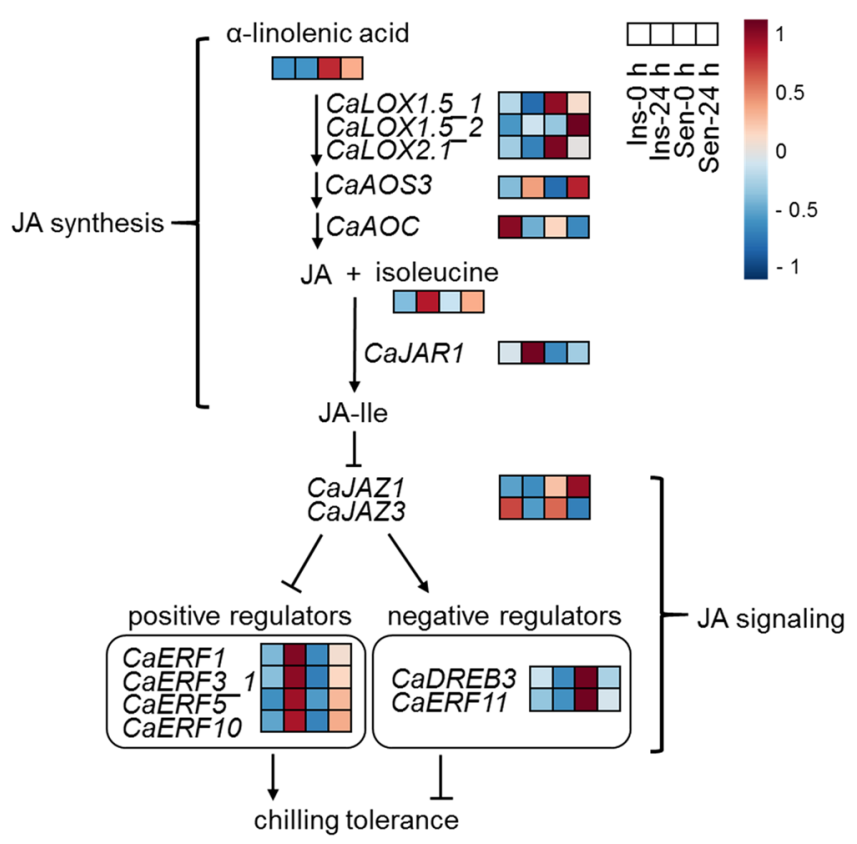

Figure 6. Putative chilling response pathway predicted in Capsicum annuum based on RNA-sequencing data. Chilling insensitive (Ins) 'UZB-GJG-1999-51' and chilling sensitive (Sen) 'C00562' peppers were treated with a chilling temperature of $2{ }^{\circ} \mathrm{C}$ for $0 \mathrm{~h}$ and $24 \mathrm{~h}$ after harvest, respectively.

temperature of $1^{\circ} \mathrm{C}^{27}$. Pyruvic acid increases the activity of alternative oxidase (AOX) within the cells ${ }^{28}$. Increased AOX gene expressions and AOX protein content then scavenges reactive oxygen species (ROS) generated under chilling conditions, resulting in higher chilling tolerance in the plant ${ }^{4}$. We speculate that the increased level of pyruvic acid in chilling-sensitive 'C00562' peppers after chilling treatment is probably due to the faster and more sensitive response to chilling stress compared to chilling-insensitive ' $U Z B-G J G-1999-51$ ' peppers.

Increased pyruvic acid has been known to accelerate amino acid synthesis ${ }^{29}$. Among amino acids, BCAAs such as isoleucine, leucine, and valine are known to control abiotic stress responses induced by chilling ${ }^{30}$ through the regulation of osmotic pressure ${ }^{31}$. BCAA levels of leucine, isoleucine, and valine in rice seedlings increased to $6.8,2.6$, and 4.5 times higher than the initial level, respectively, after a chilling treatment at $4{ }^{\circ} \mathrm{C}$ for $24 \mathrm{~h}^{32}$. In this study, BCAA levels were significantly increased in seeds of pepper fruit treated with a chilling temperature of $2{ }^{\circ} \mathrm{C}$ at $24 \mathrm{~h}$, regardless of the pepper genotype, and the increased levels were the same in both pepper genotypes. A difference in BCAA levels according to the chilling sensitivity of pepper fruit was not observed.

Similar to the result of the pyruvic acid analysis, palmitic acid, a major saturated fatty acid in pepper seeds, was significantly higher in chilling-sensitive 'C00562' than in chilling-insensitive ' $U Z B-G J G-1999-51$ ', regardless of the chilling treatment (Fig. 2B). Also, it was only significantly increased in chilling-sensitive 'C00562' after the chilling treatment, but not in chilling-insensitive 'UZB-GJG-1999-51'. Under chilling conditions, unsaturated fatty acids in cell membranes are usually converted to saturated fatty acids through the lipid peroxidation process by $\mathrm{ROS}^{33}$. In a comparison of pepper genotypes, chilling-insensitive ' $U Z B-G J G-1999-51$ ' peppers are thought to be less prone to lipid peroxidation in chilling conditions because the palmitic acid level did not change even after the chilling treatment (Fig. 2B). On the other hand, chilling-sensitive 'C00562' has a rapid lipid peroxidation process as shown by a higher level of palmitic acid after chilling than chilling-insensitive ' $U Z B-G J G-1999-51$ '. The difference in the degree of lipid peroxidation between the two genotypes might be a major factor in determining the chilling sensitivity of the two genotypes. In addition to the metabolite analysis, transcriptome analysis was performed to confirm a specific chilling response mechanism in pepper seeds.

Through transcriptome analysis of seeds obtained from chilling-insensitive and sensitive peppers treated at $2{ }^{\circ} \mathrm{C}$ for $24 \mathrm{~h}$, a total of 84,627 contigs were identified. Our results of multivariate analysis using all contigs (Supplementary Fig. S2) and hierarchical analysis (Supplementary Fig. S3) show clear separations by pepper genotypes as well as by the chilling treatment. The effect of chilling treatment could be confirmed more reliably through transcriptome analysis than by metabolite analysis.

JA is involved in chilling responses by controlling ROS through $E R F$ transcription factors ${ }^{34}$. However, the period of the chilling exposure for the activation of the JA synthesis pathway varies by plant. In Arabidopsis, JA content increased by 3 times after $1.5 \mathrm{~h}$ of chilling treatment at $4{ }^{\circ} \mathrm{C}$ and JA biosynthesis genes such as LOX1_4, $A O S$, and $A O C 1 \_3$ were significantly upregulated after $1.5 \mathrm{~h}$ of the chilling treatment ${ }^{8}$. Conversely, some crops require at least $24 \mathrm{~h}$ of chilling treatment to activate the JA synthesis pathway. For example, JA content in rice seedlings was increased by only 1.1 times after 1 day and increased by 2.0 times after 3 days of chilling treatment at $4{ }^{\circ} \mathrm{C}^{35}$. In addition, under a chilling treatment of $72 \mathrm{~h}$ or more, the expression levels of $L O X, A O S$, and $A O C$ were significantly increased in Camellia japonica 'Nuccio's Bella Rossa' compared to the other cultivars ${ }^{36}$. 
In pepper fruit stored at $0{ }^{\circ} \mathrm{C}$, the expression levels of $\mathrm{CaLOX}, \mathrm{CaAOS}$, and $\mathrm{CaAOC}$ were not different until 7 days of storage, but they were significantly different compared to the control after 14 days ${ }^{37}$. Similarly, in an experiment with $\mathrm{C}$. annuum 'Cheongyang' stored at $2{ }^{\circ} \mathrm{C}$ for 25 days, $\mathrm{CaLOX}$ and $\mathrm{CaAOC}$ expression levels were not changed until $12 \mathrm{~h}$, but the CaAOC expression level was significantly increased after 10 days during cold storage $^{5}$. In this study, the expression levels of CaLOX1.5_2 and CaAOS3 were significantly increased after $24 \mathrm{~h}$ of the chilling treatment at $2{ }^{\circ} \mathrm{C}$ in both pepper genotypes (chilling-sensitive and insensitive peppers) (Fig. 5); however, the expression levels of $\mathrm{CaLOX} 2.1$ and $\mathrm{C} a A O C$ were significantly decreased after chilling. These results might imply that the JA synthesis pathway in peppers was not perfectly activated in our experiment condition of chilling at $2{ }^{\circ} \mathrm{C}$ for $24 \mathrm{~h}$.

The expression level of CaJAR1 synthesizing JA-Ile, an active form of JA, was significantly increased in chilling-insensitive peppers but slightly increased in chilling-sensitive peppers (Fig. 5). Similarly, in Arabidopsis, the expression level of JAR1 was increased by 8.1 times after chilling treatment at $4{ }^{\circ} \mathrm{C}$ for $24 \mathrm{~h}$ compared to before the treatment ${ }^{8}$. JAR1 was more rapidly upregulated than LOX1 and AOC in Artemisia annua treated with chilling at $4{ }^{\circ} \mathrm{C}^{38}$. In summary, we could assume that expression levels of CaJAR1 in chilling-insensitive and sensitive peppers are very similar before chilling treatment but once pepper fruits are exposed to chilling, chilling-insensitive peppers increase the synthesis of JA-Ile, an active form of JA, by up-regulating CaJAR1 in response to chilling stress.

Among the ERF family genes regulated by JA signaling, the CaERF1,CaERF3_1, CaERF5, and CaERF10 expression levels were significantly increased by chilling treatment and found to be more significantly increased in chilling-insensitive genotypes. On the contrary, the expression levels of CaERF11 and CaDREB3 were significantly decreased in both genotypes by the chilling treatment (Fig. 5). ERF1, ERF3, and ERF5 have been known to increase their expression levels under chilling stress in Arabidopsis ${ }^{39}$ and cotton ${ }^{40}$. In banana, MaERF10 positively regulates chilling tolerance ${ }^{41}$. DREB3 is also known to increase chilling tolerance in tomato ${ }^{42,43}$, but in this study we obtained the opposite result for $C a D R E B 3$. Therefore, further studies are needed to confirm whether the expression pattern of $C A D R E B 3$ is specific to pepper fruit.

The involvement of ERF11 in chilling responses has not been well reported. ERF11 has been known to promote internode growth by activating gibberellin ${ }^{44}$ or to inhibit a stress response mechanism by antagonistically regulating ERF6 in the plant ${ }^{45}$. CaERF11 could be expected to play a role in suppressing the chilling tolerance of pepper fruit (Fig. 6).

In conclusion, the harvested pepper fruit in our study showed seed browning, which is a typical chilling symptom during cold storage or market distribution. Seed browning rates varied greatly according to pepper genotypes from chilling-insensitive $(0.0 \%)$ to chilling-sensitive (over $60.0 \%)$. Integrating transcriptomic and metabolomics analysis clearly revealed differential metabolite accumulations and gene expressions between the chilling-insensitive and sensitive pepper genotypes. CaERF1, CaERF3_1, CaERF5, and CaERF10 were found to be the key genes positively contributing to the chilling tolerance of insensitive pepper genotypes by showing significantly increased expressions under chilling conditions. However, CaDREB3 and CaERF11 always showed significantly higher expressions and were therefore found to be the key genes negatively contributing to the chilling tolerance of chilling-sensitive pepper genotypes. To the best of our knowledge, this study is the first to report on the molecular mechanisms regulating seed browning occurrence in C. annuum pepper fruit. These results can be used as a foundation for breeding pepper cultivars with high postharvest quality that show resistance to chilling stress. However, further research is required to confirm whether these ERE genes play a regulatory role in the chilling sensitivity of peppers, by up- or downregulating key genes.

\section{Materials and methods}

Plant materials and chilling treatment. We obtained seeds from 36 genotypes of C. annuum from the National Agrobiodiversity Center, Jeonju, Korea (Supplementary Table S1). Pepper seeds were planted in a greenhouse in Suwon, Korea. The plants were managed according to standard practices and fruits were harvested at maturity, approximately 45-50 days after full bloom, depending on the genotype. Pepper fruits of uniform size and color were harvested by hand for the experiment. They were immediately transported to the laboratory and then precooled at $18^{\circ} \mathrm{C}$ for $8 \mathrm{~h}$. After precooling, the fruits were transferred to storage chambers for cold storage at $2{ }^{\circ} \mathrm{C}$ for 3 weeks under $90 \%$ relative humidity and dark conditions.

Chilling injury symptoms in each genotype were carefully observed and photographed after cutting each fruit in half, lengthwise. This was done weekly during cold storage at $2{ }^{\circ} \mathrm{C}$ for 3 weeks. The seed browning rate of each fruit was calculated by the following equation and an average rate of seed browning for each genotype was determined. Twenty fruits were used as biological replicates in each pepper genotype.

Seed browning rate of a pepper $(\%)=[$ the number of browned seeds/the number of total (normal + browned) seeds $] \times 100$

For transcriptome and metabolomics studies, we selected two different pepper genotypes to find key genes involved in a chilling-induced seed browning based on our screening data of seed browning rates (Supplementary Table S1). The variety ' $U Z B-G J G-1999-51$ ' was selected as a chilling-insensitive pepper (seed browning rate $0.00 \%$ ) and ' $C 00562$ ' as a chilling-sensitive pepper (seed browning rate 63.86\%). Both ' $U Z B-G J G-1999-51$ ' and ' $C 00562^{\prime}$ ' fruits were exposed at $2{ }^{\circ} \mathrm{C}$ for $24 \mathrm{~h}$ without cold storage for 3 weeks. Pepper fruits were sampled before chilling treatment at $2{ }^{\circ} \mathrm{C}(0 \mathrm{~h})$ and after chilling treatment for $24 \mathrm{~h}$ at $2{ }^{\circ} \mathrm{C}(24 \mathrm{~h})$. All seeds obtained were collected without placenta, then immediately frozen in liquid nitrogen and kept at $-80^{\circ} \mathrm{C}$ for all experiments.

For further verifying our results by qPCR analysis, two other chilling-insensitive peppers, 'Takanotsume' (seed browning rate $0.00 \%$ ) and 'Hungarian Wax' (seed browning rate $4.31 \%$ ); as well as two other chilling-sensitive peppers, 'Chili bangi' (seed browning rate $41.01 \%$ ) and 'Gyeonggiyangpyeong' (seed browning rate 30.88\%) (Supplementary Table S1) were also examined. They were sampled before the chilling treatment at $2{ }^{\circ} \mathrm{C}(0 \mathrm{~h})$ and after 
the chilling treatment at $2{ }^{\circ} \mathrm{C}(24 \mathrm{~h})$. All seeds obtained were also collected without placenta, then immediately frozen in liquid nitrogen and kept at $-80^{\circ} \mathrm{C}$ for the qPCR analysis.

Chemical reagents. All chemicals were obtained from Sigma-Aldrich (St. Louis, MO, USA) or Becton Dickinson (San Jose, CA, USA). All solvents used for HPLC were purchased from J.T. Baker (Center Valley, PA, USA).

Free amino acids analysis. Free amino acids were analyzed following a previously described method ${ }^{5,46}$ with some modifications. First, frozen pepper seeds were completely ground into a fine powder using a mortar and pestle in liquid nitrogen. Then, $1.2 \mathrm{~mL}$ of $5 \%$ trichloroacetic acid was added to $100 \mathrm{mg}$ of pepper seed powder and the mixture was sonicated at room temperature for $30 \mathrm{~min}$. After reaching a concentration of $16,000 \times g$ at $4{ }^{\circ} \mathrm{C}, 1 \mathrm{~mL}$ of the supernatant was collected and filtered through a $0.45 \mu \mathrm{m}$ polyvinylidene fluoride membrane filter. After mixing $5 \mu \mathrm{L}$ of $0.4 \mathrm{~N}$ borate buffer $(\mathrm{pH} 10.2)$ and $1 \mu \mathrm{L}$ of sample, $1 \mu \mathrm{L}$ of $o$-phthalaldehyde, and 1 $\mu \mathrm{L}$ of fluorenylmethyloxycarbonyl were added for derivatization. Finally, $64 \mu \mathrm{L}$ of distilled water was added and analyzed by HPLC. The column was equipped with a Zorbax eclipse AAA $(4.6 \times 150 \mathrm{~mm}$, Agilent, Santa Clara, $\mathrm{CA}, \mathrm{USA}$ ) and the flow rate was set to $2 \mathrm{~mL} \mathrm{~min}{ }^{-1}$. The mobile phase A was set to $40 \mathrm{mM} \mathrm{NaH}_{2} \mathrm{PO}_{4}$ (pH 7.8), and $\mathrm{B}$ was set to acetonitrile:methanol: $\mathrm{H}_{2} \mathrm{O}(45: 45: 10$, v:v:v).

Fatty acid analysis. Fatty acids were analyzed following a previously described method ${ }^{47}$ with some modifications. First, $100 \mathrm{mg}$ of ground frozen pepper seed was placed in a Teflon cap tube and extracted with $2 \mathrm{~mL}$ of methylation mixture (methanol:benzene:2,2'-dimethoxypropane: $\mathrm{H}_{2} \mathrm{SO}_{4}=39: 20: 5: 2$, v:v:v:v) and $1 \mathrm{~mL}$ of heptane at $80^{\circ} \mathrm{C}$ for $2 \mathrm{~h}$. After cooling at room temperature, the supernatant was taken and analyzed by GC. Agilent 7890A (Agilent, Santa Clara, CA, USA) was used and the column was DB-23 $23(0.25 \mathrm{~mm} \times 60 \mathrm{~m} \times 0.25 \mu \mathrm{m}$, Agilent, Santa Clara, CA, USA). The FID detector was set at $280{ }^{\circ} \mathrm{C}$ and the flow rates were $35 \mathrm{~mL} \mathrm{~min}^{-1}$ for $\mathrm{H}_{2}$, $350 \mathrm{~mL} \mathrm{~min}^{-1}$ for air, and $35 \mathrm{~mL} \mathrm{~min}^{-1}$ for He. The injector temperature was set at $250^{\circ} \mathrm{C}$. The oven temperature increased from $50{ }^{\circ} \mathrm{C}$ to $130^{\circ} \mathrm{C}$ by $15^{\circ} \mathrm{C} \mathrm{m^{-1 }}$, to $170{ }^{\circ} \mathrm{C}$ by $8{ }^{\circ} \mathrm{C} \mathrm{min}-1$, and to $215^{\circ} \mathrm{C}$ by $2{ }^{\circ} \mathrm{C} \mathrm{min}{ }^{-1}$.

Untargeted polar phase metabolite analysis. Polar phase compounds were extracted following a previously described method ${ }^{5,48,49}$ with some modifications. Fifty-mg of ground frozen pepper seeds was mixed with $1.2 \mathrm{~mL}$ of methanol and shaken at $75^{\circ} \mathrm{C}$ for $30 \mathrm{~min}$; then centrifuged at $13,500 \times g$ for $10 \mathrm{~min}$. Next, $0.7 \mathrm{~mL}$ of the supernatant was transferred to a $2 \mathrm{~mL}$-microtube and mixed with $0.5 \mathrm{~mL}$ of chloroform and $20 \mu \mathrm{L}$ of ribitol (internal standard). Immediately after adding $0.7 \mathrm{~mL}$ of distilled water, it was centrifuged at $2500 \times g$ for $10 \mathrm{~min}$. Then $0.5 \mathrm{~mL}$ of the supernatant was concentrated using a nitrogen evaporator (MG-2200, Eyela, Japan). Methoxyamine hydrochloride $(50 \mu \mathrm{L})$ was added and incubated at $37^{\circ} \mathrm{C}$ for $2 \mathrm{~h}$. After incubation, $40 \mu \mathrm{L}$ of sample and $100 \mu \mathrm{L}$ of $N$-methyl- $N$-trifluoroacetamide were mixed and incubated at $37^{\circ} \mathrm{C}$ for $30 \mathrm{~min}$. Then, $1 \mu \mathrm{L}$ of the sample was injected into the GC-MS ISQ LT system (Thermo Fisher Scientific, Waltham, MA, USA) using an auto sampler. The DB-5-fused silica capillary column $(0.25 \mathrm{~mm} \times 30 \mathrm{~m} \times 0.25 \mu \mathrm{m}$, Agilent, Santa Clara, CA, USA) was used and the oven temperature was set to increase from $50{ }^{\circ} \mathrm{C}$ to $310^{\circ} \mathrm{C}$ at a rate of $5{ }^{\circ} \mathrm{C} \mathrm{min}^{-1}$. The injector was in the split-less mode at $250^{\circ} \mathrm{C}$. Helium was used as the carrier gas at a flow rate of $1 \times 10^{-3} \mathrm{~L} \mathrm{~min}^{-1}$. The range of mass scan was from 35 to $550 \mathrm{~m} / \mathrm{z}$.

Total RNA isolation, cDNA library construction, and sequencing. Frozen seeds were ground into a fine powder using a mortar and pestle in liquid nitrogen and $100 \mathrm{mg}$ of powder was used for total RNA extraction. Total RNA was extracted using Ribospin Seed/Fruit Kit (GeneAll, Seoul, Korea) following the manufacturer's instructions. Extracted total RNA was used for RNA-seq and cDNA synthesis. cDNA was synthesized using an amfiRivert Platinum cDNA Synthesis Master Mix Kit (GenDEPOT, Baker, TX, USA) following the manufacturer's instructions.

Transcriptome analysis by RNA-Seq. Total RNA from 'UZB-GJG-1999-51' and 'C00562' peppers treated with chilling for $0 \mathrm{~h}$ and $24 \mathrm{~h}$ were used for RNA-seq. RNA-seq was performed using the Illumina Hi-Seq 2500 system (Illumina, San Diego, CA, USA) with 151-bp paired-end at the National Instrumentation Center for Environmental Management (NICEM), Seoul National University, South Korea. As a pretreatment process, artifacts such as adaptor sequence, contaminant DNA, and PCR duplicates, were removed from raw reads. Then, preprocessed reads were assembled and mapped to Pepper Zunla 1 Ref_v1.0 reference genome (GCF_000710875.1, NCBI).

Identification of DEGs. To identify DEGs between the two different genotypes, 'UZB-GJG-1999-51' and 'C00562' pepper fruits were treated with chilling for $0 \mathrm{~h}$ or $24 \mathrm{~h}$, at $2{ }^{\circ} \mathrm{C}$. The expression levels of each read were calculated according to the fragment per kilobase of transcript per million mapped reads (FPKM) value. DEGs were selected using a threshold of $\geq$ twofold upregulated or downregulated genes with an FDR $<0.05$ and all processes were performed by EdgeR software.

GO term and KEGG enrichment analyses for DEGs. Functional-enrichment analysis including GO and KEGG were performed using Blast2GO 5.2 $2^{50}$ and MapMan 3.6.0 (https://mapman.gabipd.org), respectively.

qPCR analysis. For qPCR, the cDNA was diluted tenfold. The qPCR was performed using 2X Real-Time PCR Master Mix (Biofact, Daejeon, Korea) in a final volume of $20 \mu \mathrm{L}$ and CFX Connect Real-Time System (BIO- 
RAD, Hercules, CA, USA) under the following conditions: $95^{\circ} \mathrm{C}$ for $15 \mathrm{~min}$ followed by 40 cycles of $95{ }^{\circ} \mathrm{C}$ for $20 \mathrm{~s}, 55^{\circ} \mathrm{C}$ for $40 \mathrm{~s}$, and $72^{\circ} \mathrm{C}$ for $20 \mathrm{~s}$. Relative gene expressions were determined with normalization against the expression of the pepper CaActin7 gene. The primers used for the qPCR are listed in Supplementary Table S2, and are designed based on a reference gene set using the Primer 3 plus server (https://www.bioinformatics.nl/ cgi-bin/primer3plus/primer3plus.cgi). The relative gene expression was calculated using the $2^{-\Delta \Delta \mathrm{Ct}} \mathrm{method}^{51}$.

Statistical analysis. All experimental analyses were conducted in randomized designs with three biological replicates $(n=3)$. Statistical comparison between the means of the experimental groups was carried out using SPSS ver. 22.0 (IBM, Armonk, NY, USA). One-way analysis of variance and Duncan's multiple range test were performed to determine significant differences. The metabolite data were auto-scaled and used for PCA, PLSDA, heat-map analysis, and metabolite enrichment analysis with MetaboAnalyst 3.0 software (www.metaboanal yst.ca), and PPI analysis and correlation network analysis with Cytoscape v3.6.1 software (https://cytoscape. github.io/).

\section{Data availability}

All datasets generated for this study are included in the article and Supplementary Materials.

Received: 13 July 2020; Accepted: 9 October 2020

Published online: 21 October 2020

\section{References}

1. Özden, Ç. \& Bayindirli, L. Effects of combinational use of controlled atmosphere, cold storage and edible coating applications on shelf life and quality attributes of green peppers. Eur. Food Res. Technol. 214, 320-326 (2002).

2. Valenzuela, J. L. et al. Oxidative stress associated with chilling injury in immature fruit: postharvest technological and biotechnological solutions. Int. J. Mol. Sci. 18, 1467 (2017).

3. Boonsiri, K., Ketsa, S. \& van Doorn, W. G. Seed browning of hot peppers during low temperature storage. Postharvest Biol. Technol. 45, 358-365 (2007).

4. Fung, R. W. M., Wang, C. Y., Smith, D. L., Gross, K. C. \& Tian, M. MeSA and MeJA increase steady-state transcript levels of alternative oxidase and resistance against chilling injury in sweet peppers (Capsicum annuum L.). Plant Sci. 166, 711-719 (2004).

5. Seo, J., Yi, G., Lee, J. G., Choi, J. H. \& Lee, E. J. Seed browning in pepper (Capsicum annuum L.) fruit during cold storage is inhibited by methyl jasmonate or induced by methyl salicylate. Postharvest Biol. Technol. 166, 111210 (2020).

6. Fonseca, S. et al. (+)-7-iso-Jasmonoyl-L-isoleucine is the endogenous bioactive jasmonate. Nat. Chem. Biol. 5, 344-350 (2009).

7. Hu, Y. et al. Jasmonate regulates leaf senescence and tolerance to cold stress: crosstalk with other phytohormones. J. Exp. Bot. 68, 1361-1369 (2017).

8. Hu, Y., Jiang, L., Wang, F. \& Yu, D. Jasmonate regulates the inducer of CBF expression-c-repeat binding factor/DRE binding factor 1 cascade and freezing tolerance in Arabidopsis. Plant Cell. 25, 2907-2924 (2013).

9. Wang, F. et al. Phytochrome A and B function antagonistically to regulate cold tolerance via abscisic acid-dependent jasmonate signaling. Plant Cell Physiol. 170, 459-471 (2016).

10. Pratiwi, P. et al. Identification of jasmonic acid and jasmonoyl-isoleucine, and characterization of AOS, AOC, OPR and JAR1 in the model lycophyte Selaginella moellendorffii. Plant Cell Physiol. 58, 789-801 (2017).

11. Zhang, F. et al. Structural basis of JAZ repression of MYC transcription factors in jasmonate signalling. Nature 525, 269-273 (2015).

12. Pieterse, C. M., Pierik, R. \& Van Wees, S. C. Different shades of JAZ during plant growth and defense. New Phytol. 204, 261-264 (2014).

13. Pauwels, L. \& Goossens, A. The JAZ proteins: a crucial interface in the jasmonate signaling cascade. Plant Cell. 23, 3089-3100 (2011).

14. Melotto, M. et al. A critical role of two positively charged amino acids in the Jas motif of Arabidopsis JAZ proteins in mediating coronatine-and jasmonoyl isoleucine-dependent interactions with the COI1 F-box protein. Plant J. 55, 979-988 (2008).

15. Lorenzo, O., Piqueras, R., Sánchez-Serrano, J. J. \& Solano, R. ETHYLENE RESPONSE FACTOR1 integrates signals from ethylene and jasmonate pathways in plant defense. Plant Cell. 15, 165-178 (2003).

16. Sears, M. T. et al. NtERF32: a non-NIC2 locus AP2/ERF transcription factor required in jasmonate-inducible nicotine biosynthesis in tobacco. Plant Mol. Biol. 84, 49-66 (2014).

17. Pré, M. et al. The AP2/ERF domain transcription factor ORA59 integrates jasmonic acid and ethylene signals in plant defense. Plant Physiol. 147, 1347-1357 (2008).

18. Xie, Z., Nolan, T. M., Jiang, H. \& Yin, Y. AP2/ERF transcription factor regulatory networks in hormone and abiotic stress responses in Arabidopsis. Front. Plant Sci. 10, 228 (2019).

19. Bolt, S., Zuther, E., Zintl, S., Hincha, D. K. \& Schmülling, T. ERF105 is a transcription factor gene of Arabidopsis thaliana required for freezing tolerance and cold acclimation. Plant Cell Environ. 40, 108-120 (2017).

20. Wang, M. et al. ERF109 of trifoliate orange (Poncirus trifoliata (L.) Raf.) contributes to cold tolerance by directly regulating expression of Prx1 involved in antioxidative process. Plant Biotechnol. J. 17, 1316-1332 (2019).

21. Sun, X. et al. The ethylene response factor VaERF 092 from Amur grape regulates the transcription factor VaWRKY 33, improving cold tolerance. Plant J. 99, 988-1002 (2019).

22. Lv, K. et al. Overexpression of an $A P 2 / E R F$ family gene, $B P E R F 13$, in birch enhances cold tolerance through upregulating $C B F$ genes and mitigating reactive oxygen species. Plant Sci. 292, 110375 (2020).

23. Leone, M., Keller, M. M., Cerrudo, I. \& Ballaré, C. L. To grow or defend? Low red: far-red ratios reduce jasmonate sensitivity in Arabidopsis seedlings by promoting DELLA degradation and increasing JAZ10 stability. New Phytol. 204, 355-367 (2014).

24. Lissarre, M., Ohta, M., Sato, A. \& Miura, K. Cold-responsive gene regulation during cold acclimation in plants. Plant Signal. Behav. 5, 948-952 (2010).

25. Fiehn, O. et al. Metabolite profiling for plant functional genomics. Nat. Biotechnol. 18, 1157-1161 (2000).

26. Lin, W., Li, Y., Lu, Q., Lu, H. \& Li, J. Combined analysis of the metabolome and transcriptome identified candidate genes involved in phenolic acid biosynthesis in the leaves of Cyclocarya paliurus. Int. J. Mol. Sci. 21, 1337 (2020).

27. Tsuchida, H., Dan-Hong, C., Inoue, K., Kozukue, N. \& Mizuno, S. Changes in pyruvic acid content and GPT activity in chillingsensitive and nonsensitive crops. HortSci. 25, 952-953 (1990).

28. Kumar, N., Vyas, D. \& Kumar, S. Plants at high altitude exhibit higher component of alternative respiration. J. Plant Physiol. 164, 31-38 (2007).

29. Vanlerberghe, G. C. \& McIntosh, L. Alternative oxidase: from gene to function. Annu. Rev. Plant Physiol. Plant Mol. Biol. 48, 703-734 (1997). 
30. Obata, T. \& Fernie, A. R. The use of metabolomics to dissect plant responses to abiotic stresses. Cell. Mol. Life Sci. 69, 3225-3243 (2012).

31. Joshi, V., Joung, J. G., Fei, Z. \& Jander, G. Interdependence of threonine, methionine and isoleucine metabolism in plants: accumulation and transcriptional regulation under abiotic stress. Amino Acids 39, 933-947 (2010).

32. Zhao, X. Q. et al. Temporal profiling of primary metabolites under chilling stress and its association with seedling chilling tolerance of rice (Oryza sativa L.). Rice. 6, 23 (2013).

33. Liavonchanka, A. \& Feussner, I. Lipoxygenases: occurrence, functions and catalysis. J. Plant Physiol. 163, 348-357 (2006).

34. Sharma, M. \& Laxmi, A. Jasmonates: emerging players in controlling temperature stress tolerance. Front. Plant Sci. 6, 1129 (2015).

35. Du, H., Liu, H. \& Xiong, L. Endogenous auxin and jasmonic acid levels are differentially modulated by abiotic stresses in rice. Front. Plant Sci. 4, 397 (2013).

36. Li, Q. et al. RNA-seq based transcriptomic analysis uncovers $\alpha$-linolenic acid and jasmonic acid biosynthesis pathways respond to cold acclimation in Camellia japonica. Sci. Rep. 6, 1-13 (2016).

37. Shin, S. Y., Park, M. H., Choi, J. W. \& Kim, J. G. Gene network underlying the response of harvested pepper to chilling stress. J. Plant Physiol. 219, 112-122 (2017).

38. Liu, W. et al. Cold stress improves the production of artemisinin depending on the increase in endogenous jasmonate. Biotechnol. Appl. Biochem. 64, 305-314 (2017).

39. Qiao, Z. X., Huang, B. \& Liu, J. Y. Molecular cloning and functional analysis of an ERF gene from cotton (Gossypium hirsutum). BBA Gene Regul. Mech. 1779, 122-127 (2008).

40. Jin, L. G., Li, H. \& Liu, J. Y. Molecular characterization of three ethylene responsive element binding factor genes from cotton. J. Integr. Plant Biol. 52, 485-495 (2010).

41. Qi, X. N. et al. A banana fruit transcriptional repressor MaERF10 interacts with MaJAZ3 to strengthen the repression of JA biosynthetic genes involved in MeJA-mediated cold tolerance. Postharvest Biol. Technol. 120, 222-231 (2016).

42. Islam, M. S. \& Wang, M. H. Expression of dehydration responsive element-binding protein-3 (DREB3) under different abiotic stresses in tomato. BMB Rep. 42, 611-616 (2009).

43. Wang, G. et al. A tomato transcription factor, SIDREB3 enhances the tolerance to chilling in transgenic tomato. Plant Physiol. Biochem. 142, 254-262 (2019).

44. Zhou, X. et al. The ERF11 transcription factor promotes internode elongation by activating gibberellin biosynthesis and signaling. Plant Physiol. 171, 2760-2770 (2016).

45. Dubois, M. et al. The ETHYLENE RESPONSE FACTORs ERF6 and ERF11 antagonistically regulate mannitol-induced growth inhibition in Arabidopsis. Plant Physiol. 169, 166-179 (2015).

46. Park, J. H., Park, J. S. \& Choi, J. S. Basic amino acid-conjugated polyamidoamine dendrimers with enhanced gene transfection efficiency. Macromol. Res. 22, 500-508 (2014).

47. Garcés, R. \& Mancha, M. One-step lipid extraction and fatty acid methyl esters preparation from fresh plant tissues. Anal. Biochem. 211, 139-143 (1993).

48. Lim, S., Lee, J. G. \& Lee, E. J. Comparison of fruit quality and GC-MS-based metabolite profiling of kiwifruit 'Jecy green': natural and exogenous ethylene-induced ripening. Food Chem. 234, 81-92 (2017).

49. Bang, J., Lim, S., Yi, G., Lee, J. G. \& Lee, E. J. Integrated transcriptomic-metabolomic analysis reveals cellular responses of harvested strawberry fruit subjected to short-term exposure to high levels of carbon dioxide. Postharvest Biol. Technol. 148, 120-131 (2019).

50. Conesa, A. et al. Blast2GO: a universal tool for annotation, visualization and analysis in functional genomics research. Bioinformatics 21, 3674-3676 (2005).

51. Livak, K. J. \& Schmittgen, T. D. Analysis of relative gene expression data using real-time quantitative PCR and the 2(-Delta Delta C(T)) method. Methods 25, 402-408 (2001).

\section{Acknowledgements}

This work was supported by the Basic Science Research Program through the National Research Foundation [NRF, 2016R1A1A1A05919210, 2020R1F1A1068842 to E.J.L.] of Korea funded by the Ministry of Education, Science, and Technology and the Korea Institute of Planning and Evaluation for Technology in Food, Agriculture, and Forestry [IPET, 617068-05-1-WT111 to E.J.L.), Republic of Korea.

\section{Author contributions}

Study concepts: J.G.L., E.J.L, Study design: J.G.L., E.J.L., G.Y., Data acquisition and analysis: J.G.L., J.S., Manuscript preparation: J.G.L., E.J.L., Manuscript editing and review: G.Y., B.C.K., J.H.C., and E.J.L.

\section{Competing interests}

The authors declare no competing interests.

\section{Additional information}

Supplementary information is available for this paper at https://doi.org/10.1038/s41598-020-75055-z.

Correspondence and requests for materials should be addressed to E.J.L.

Reprints and permissions information is available at www.nature.com/reprints.

Publisher's note Springer Nature remains neutral with regard to jurisdictional claims in published maps and institutional affiliations.

(c) (i) Open Access This article is licensed under a Creative Commons Attribution 4.0 International License, which permits use, sharing, adaptation, distribution and reproduction in any medium or format, as long as you give appropriate credit to the original author(s) and the source, provide a link to the Creative Commons licence, and indicate if changes were made. The images or other third party material in this article are included in the article's Creative Commons licence, unless indicated otherwise in a credit line to the material. If material is not included in the article's Creative Commons licence and your intended use is not permitted by statutory regulation or exceeds the permitted use, you will need to obtain permission directly from the copyright holder. To view a copy of this licence, visit http://creativecommons.org/licenses/by/4.0/.

(C) The Author(s) 2020 